\title{
Acoustic Streaming and Its Suppression in Inhomogeneous Fluids
}

\author{
Karlsen, Jonas Tobias; Qiu, Wei; Augustsson, Per; Bruus, Henrik
}

Published in:

Physical Review Letters

Link to article, DOI:

10.1103/PhysRevLett.120.054501

Publication date:

2018

Document Version

Publisher's PDF, also known as Version of record

Link back to DTU Orbit

Citation (APA):

Karlsen, J. T., Qiu, W., Augustsson, P., \& Bruus, H. (2018). Acoustic Streaming and Its Suppression in Inhomogeneous Fluids. Physical Review Letters, 120(5), [054501].

https://doi.org/10.1103/PhysRevLett.120.054501

\section{General rights}

Copyright and moral rights for the publications made accessible in the public portal are retained by the authors and/or other copyright owners and it is a condition of accessing publications that users recognise and abide by the legal requirements associated with these rights.

- Users may download and print one copy of any publication from the public portal for the purpose of private study or research.

- You may not further distribute the material or use it for any profit-making activity or commercial gain

- You may freely distribute the URL identifying the publication in the public portal

If you believe that this document breaches copyright please contact us providing details, and we will remove access to the work immediately and investigate your claim. 


\title{
Acoustic Streaming and Its Suppression in Inhomogeneous Fluids
}

\author{
Jonas T. Karlsen, ${ }^{1, *}$ Wei Qiu, ${ }^{1}$ Per Augustsson, ${ }^{2}$ and Henrik Bruus ${ }^{1, \dagger}$ \\ ${ }^{1}$ Department of Physics, Technical University of Denmark, DTU Physics Building 309, DK-2800 Kongens Lyngby, Denmark \\ ${ }^{2}$ Department of Biomedical Engineering, Lund University, Ole Römers väg 3, 22363 Lund, Sweden
}

(Received 23 July 2017; revised manuscript received 11 December 2017; published 30 January 2018)

\begin{abstract}
We present a theoretical and experimental study of boundary-driven acoustic streaming in an inhomogeneous fluid with variations in density and compressibility. In a homogeneous fluid this streaming results from dissipation in the boundary layers (Rayleigh streaming). We show that in an inhomogeneous fluid, an additional nondissipative force density acts on the fluid to stabilize particular inhomogeneity configurations, which markedly alters and even suppresses the streaming flows. Our theoretical and numerical analysis of the phenomenon is supported by ultrasound experiments performed with inhomogeneous aqueous iodixanol solutions in a glass-silicon microchip.
\end{abstract}

DOI: 10.1103/PhysRevLett.120.054501

Acoustic streaming is the steady vortical flow that accompanies the propagation of acoustic waves in viscous fluids. This ubiquitous phenomenon [1,2], studied as early as 1831 by Faraday observing the motion of powder above a vibrating Chladni plate [3], is driven by a nonzero divergence in the nonlinear momentum-flux-density tensor. In a homogeneous fluid, this divergence is caused by two acoustic energy dissipation mechanisms. One mechanism is dissipation in the thin boundary layers, where the acoustic fluid velocity changes to match the velocity of the boundary. The resulting streaming, called boundarydriven Rayleigh streaming [4,5], is typically observed in standing wave fields near walls [6] or suspended objects [7]. The other mechanism is the attenuation of acoustic waves in the bulk of the fluid, which produces streaming known as bulk-driven Eckart streaming [8], typically observed in systems much larger than the wavelength [9]. Both cases have been extensively studied theoretically [10-13], and the phenomenon continues to attract attention due to its importance in thermoacoustic engines [14-16], ultrasound contrast agents, sonoporation, and drug delivery [17-19], and the manipulation of particles and cells in microscale acoustofluidics [20-27].

Recent experiments on fluids have revealed that inhomogeneities in density $\rho_{0}$ and compressibility $\kappa_{0}$, introduced by a solute concentration field, can be acoustically relocated into stabilized configurations [28,29]. In subsequent work [30,31], we showed that fast-time-scale acoustics in such inhomogeneous fluids spawns a timeaveraged acoustic force density $\boldsymbol{f}_{\text {ac }}$ acting on the fluid on the slower hydrodynamic time scale, and that $f_{\text {ac }}$ leads to the observed relocation and stabilization of inhomogeneities. The experiments also indicated that boundary-driven streaming is suppressed in inhomogeneous fluids [29], and we hypothesized that this hitherto unexplored phenomenon can be explained by $f_{\text {ac. }}$.
In this Letter, we investigate this hypothesis by combining the theories of acoustic streaming [10-13] and the acoustic force density [30]. We verify analytically the limiting cases of the combined theory, and proceed to develop a full numerical model of boundary-driven acoustic streaming in inhomogeneous viscous fluids. We know of only one similar theoretical study, which however is limited to prescribed static inhomogeneities [32]. Using our dynamic theory, we simulate the evolution of acoustic streaming, as an acoustically stabilized density profile evolves by diffusion and advection. Experimentally, we measure this evolution in an inhomogeneous aqueous iodixanol solution in an ultrasound-activated glass-silicon microchannel, Fig. 1, that enables injection of layered fluids creating a density gradient across the channel width [29,30].

Our main findings are (i) that the competition between the boundary-induced streaming stresses and the inhomogeneity-induced acoustic force density introduces a dynamic length scale $\Delta$ of the streaming vortex size, (ii) that initially $\Delta \ll \Delta_{\text {hom }} \sim \min \left\{\frac{1}{8} \lambda, \frac{1}{4} H\right\}$, where $\Delta_{\text {hom }}$

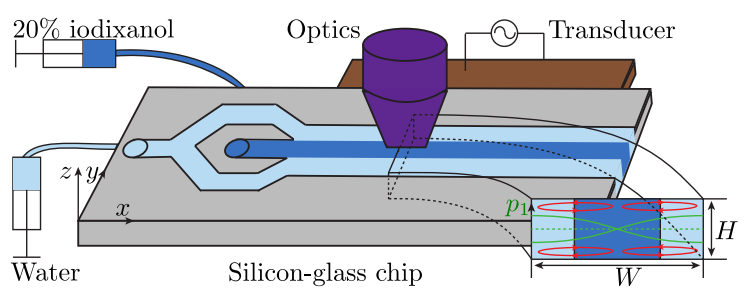

FIG. 1. Sketch of the acoustofluidic silicon chip (gray) sealed with a glass lid, which allows optical recording (purple) of the tracer bead motion (red trajectories) in the channel cross section of width $W=375 \mu \mathrm{m}$ and height $H=130 \mu \mathrm{m}$. A 20\% iodixanol solution (dark blue) is injected in the center and laminated by pure water (light blue). The piezoelectric transducer (brown) excites the resonant half-wave pressure field $p_{1}$ (inset, green) at $2 \mathrm{MHz}$. 
is the vortex size in a homogeneous fluid set by the acoustic wavelength $\lambda$ or the channel height $H$, and (iii) that in the bulk farther than $\Delta$ from the boundaries, the streaming flow is suppressed. $\Delta$ increases in time, as diffusion and advection smear the inhomogeneity, and the vortices eventually expand into the bulk, similar to homogeneous fluids. These findings are rationalized by simple scaling arguments.

Our analysis of acoustic streaming in inhomogeneous fluids elucidates fundamental physical aspects and further has potential applications in nanoparticle manipulation. Indeed, the suppression of acoustic streaming may enable the acoustophoretic manipulation of small bioparticles such as bacteria, exosomes, and viruses [33], that is otherwise hampered by the unfavorable scalings of the radiation force relative to the streaming-induced drag force with decreasing particle size [22,34]. Nanoparticle manipulation has been attempted by suppressing streaming using pulsed actuation [35,36], and by engineering streaming patterns in special geometries [37-39].

Separation of time scales. - Our analysis is based on the separation of time scales between the fast acoustics $t \sim 0.1 \mu \mathrm{s}$ and the slow hydrodynamics $\tau \sim 10 \mathrm{~ms}$ [30]. Because $\tau \sim 10^{5} t$, the acoustic fields can be computed while keeping the hydrodynamic degrees of freedom fixed at each instance in time $\tau$. Assuming the system to be timeharmonically actuated at the angular frequency $\omega$, the density $\rho$ is written as

$$
\rho=\rho_{0}(\boldsymbol{r}, \tau)+\rho_{1}(\boldsymbol{r}, \tau) e^{-i \omega t} .
$$

Here, $\rho_{0}$ is the hydrodynamic density, and $\rho_{1}$ is the acoustic perturbation.

Fast-time-scale acoustics.-Using perturbation expansions of the form (1) in the equations for conservation of fluid momentum and mass, the first-order equations for the acoustic perturbations in velocity $\boldsymbol{v}_{1}$, pressure $p_{1}$, and density $\rho_{1}$ become

$$
\begin{aligned}
-i \omega \rho_{0} \boldsymbol{v}_{1} & =\boldsymbol{\nabla} \cdot \boldsymbol{\sigma}_{1}, \\
-i \omega \kappa_{0} p_{1} & =-\boldsymbol{\nabla} \cdot \boldsymbol{v}_{1}, \\
-i \omega \rho_{0} \kappa_{0} p_{1} & =-i \omega \rho_{1}+\boldsymbol{v}_{1} \cdot \boldsymbol{\nabla} \rho_{0} .
\end{aligned}
$$

Here, $\sigma_{1}$ is the first-order fluid stress tensor, obtained by replacing $p$ by $p_{1}$ and $\boldsymbol{v}$ by $\boldsymbol{v}_{1}$ in the usual fluid stress tensor $\sigma$ [30]. The local speed of sound is $c_{0}=1 / \sqrt{\rho_{0} \kappa_{0}}$.

In viscous acoustics, the oscillation velocity $v_{1}$ attains the wall velocity on the length scale $\delta=\sqrt{2 \nu_{0} / \omega}(\approx 0.4 \mu \mathrm{m}$ for water at $2 \mathrm{MHz}$ ), where $\nu_{0}=\eta_{0} / \rho_{0}$ with $\nu_{0}$ and $\eta_{0}$ being the kinematic and dynamic viscosities, respectively. The time-averaged stress driving the streaming is generated within these narrow boundary layers. For inviscid acoustics, Eq. (2) reduces to the standard wave equation for inhomogeneous media $[40,41]$.
Slow-time-scale dynamics. - The fluid inhomogeneity is caused by a solute concentration field $s(\boldsymbol{r}, \tau)$, which is being transported on the slow time scale. This changes the hydrodynamic fluid density $\rho_{0}$, compressibility $\kappa_{0}$, and dynamic viscosity $\eta_{0}$,

$\rho_{0}=\rho_{0}[s(\boldsymbol{r}, \tau)], \quad \kappa_{0}=\kappa_{0}[s(\boldsymbol{r}, \tau)], \quad \eta_{0}=\eta_{0}[s(\boldsymbol{r}, \tau)]$.

For iodixanol, the specific dependencies in Eq. (3) are known experimentally, and $c_{0}$ is nearly independent of $s$, so that $\boldsymbol{\nabla} \kappa_{0} \approx-\left(\rho_{0} c_{0}\right)^{-2} \boldsymbol{\nabla} \rho_{0}[29,30]$.

The hydrodynamics on the slow time scale $\tau$ is governed by the momentum- and mass-continuity equations for the fluid velocity $\boldsymbol{v}(\boldsymbol{r}, \tau)$ and pressure $p(\boldsymbol{r}, \tau)$, and by the advection-diffusion equation for the concentration $s(\boldsymbol{r}, \tau)$ of the solute with diffusivity $D$ [30],

$$
\begin{aligned}
\partial_{\tau}\left(\rho_{0} \boldsymbol{v}\right) & =\boldsymbol{\nabla} \cdot\left[\boldsymbol{\sigma}-\rho_{0} \boldsymbol{v} \boldsymbol{v}\right]+\boldsymbol{f}_{\mathrm{ac}}+\rho_{0} \boldsymbol{g}, \\
\partial_{\tau} \rho_{0} & =-\boldsymbol{\nabla} \cdot\left(\rho_{0} \boldsymbol{v}\right), \\
\partial_{\tau} s & =-\boldsymbol{\nabla} \cdot[-D \boldsymbol{\nabla} s+\boldsymbol{v} s] .
\end{aligned}
$$

Here, $\boldsymbol{g}$ is the gravitational acceleration, $\boldsymbol{\sigma}$ is the fluid stress tensor, and $f_{\mathrm{ac}}$ is the acoustic force density.

All types of time-averaged acoustic flows, such as Rayleigh and Eckart streaming [10-13] and the relocation flows in inhomogeneous fluids [30,31], are driven by the divergence of the oscillation-time-averaged acoustic momentum-flux-density tensor $\left\langle\boldsymbol{\Pi}_{\mathrm{ac}}\right\rangle$. In particular [30],

$$
\boldsymbol{f}_{\mathrm{ac}}=-\boldsymbol{\nabla} \cdot\left\langle\boldsymbol{\Pi}_{\mathrm{ac}}\right\rangle \text {. }
$$

$\left\langle\boldsymbol{\Pi}_{\mathrm{ac}}\right\rangle$ is given by products of first-order acoustic fields,

$$
\begin{aligned}
& \left\langle\boldsymbol{\Pi}_{\mathrm{ac}}\right\rangle=\left\langle p_{11}\right\rangle \mathbf{1}+\left\langle\rho_{0} \boldsymbol{v}_{1} \boldsymbol{v}_{1}\right\rangle, \\
& \left\langle p_{11}\right\rangle=\frac{1}{4} \kappa_{0}\left|p_{1}\right|^{2}-\frac{1}{4} \rho_{0}\left|\boldsymbol{v}_{1}\right|^{2},
\end{aligned}
$$

where $\left\langle p_{11}\right\rangle$ is a local oscillation-time-averaged acoustic pressure, which for an inhomogeneous fluid depends on the solute concentration $s$. Combining Eqs. (5) and (6), the general expression for $f_{\text {ac }}$ in viscous inhomogeneous acoustics becomes

$$
\boldsymbol{f}_{\mathrm{ac}}=-\boldsymbol{\nabla}\left\langle p_{11}\right\rangle-\boldsymbol{\nabla} \cdot\left\langle\rho_{0} \boldsymbol{v}_{1} \boldsymbol{v}_{1}\right\rangle .
$$

Note that in Eq. (4a), $\boldsymbol{\nabla}\left\langle p_{11}\right\rangle$ from $\boldsymbol{f}_{\text {ac }}$ cannot simply be absorbed in the pressure gradient $\boldsymbol{\nabla} p$ contained in $\boldsymbol{\nabla} \cdot \boldsymbol{\sigma}$, as its explicit dependence of the dynamical variable $s$, expressed in Eq. (6b), would be lost.

Expression (7) for $f_{\text {ac }}$ may be simplified in two special cases. First, in a viscous homogeneous fluid $\left\langle p_{11}\right\rangle$ is independent of $s$, so $\boldsymbol{\nabla}\left\langle p_{11}\right\rangle$ can be absorbed into $\boldsymbol{\nabla} p$ in 
Eq. (4a) by redefining the pressure from $p$ to $\tilde{p}=p+\left\langle p_{11}\right\rangle$. Consequently,

$$
\boldsymbol{f}_{\mathrm{ac}}^{\mathrm{hom}}=-\boldsymbol{\nabla} \cdot\left\langle\rho_{0} \boldsymbol{v}_{1} \boldsymbol{v}_{1}\right\rangle .
$$

Indeed, this is how the driving terms are often presented in classical [10-12] and more recent $[6,9,42]$ works on time-independent acoustic streaming.

In the second case of inhomogeneous but inviscid acoustics, we recently demonstrated that Eq. (7) yields

$$
\boldsymbol{f}_{\mathrm{ac}}^{\text {invisc }}=-\frac{1}{4}\left|p_{1}\right|^{2} \nabla \kappa_{0}-\frac{1}{4}\left|\boldsymbol{v}_{1}\right|^{2} \nabla \rho_{0},
$$

and that this nondissipative force density is driving the slow-time-scale relocation of the fluid inhomogeneities into stable field-dependent configurations [30,31].

In the context of boundary-driven acoustic streaming in inhomogeneous fluids, the content of Eqs. (7)-(9) is as follows: in the boundary layers, the dissipation of acoustic energy leads to time-averaged stresses, confined on the length scale $\delta$, that cause boundary-driven streaming flows. However, in the presence of gradients in the density and compressibility, the nondissipative acoustic force density tends to stabilize the fluid (after initial focusing) in a certain static inhomogeneity configuration [30], thereby counteracting the advective streaming flow. While Eqs. (8) and (9) demonstrate that these two force densities are present in inhomogeneous viscous fluids, they cannot in general be separated analytically. The force density responsible for bulk-driven Eckart streaming is included in Eq. (7), but it is negligible in this study.

Numerical model in 2D.-The dynamics in the 2D channel cross section is solved numerically, under stopflow conditions with the initial condition sketched in Fig. 1, using a weak-form finite-element implementation in COMSOL Multiphysics [43] with regular rectangular mesh elements [44]. A segregated solver solves the time-dependent problem in two steps. (i) The fast-time-scale acoustics (2) in the inhomogeneous medium is solved while keeping the hydrodynamic degrees of freedom fixed. This allows computation of the time-averaged acoustic force density $f_{\text {ac }}$ (7). (ii) The slow-time-scale dynamics (4) is then integrated in time $\tau$ using a generalized alpha solver with a damping parameter of 0.25 , and a maximum time step $\Delta \tau=7.5 \mathrm{~ms}$, while keeping the acoustic energy density fixed at $E_{\mathrm{ac}}=$ $50 \mathrm{~Pa}$ [45]. This model extends our previous model [30,31] by explicitly solving for the fast-time-scale viscous acoustics in the inhomogeneous medium, a necessity for computing the boundary-layer stresses that drive streaming.

Experimental method.-The experiments were performed using a long straight microchannel of height $H=130 \mu \mathrm{m}$ and width $W=375 \mu \mathrm{m}$ in a silicon-glass chip with an attached piezoelectric transducer. A laminated flow of water and an aqueous 20\% iodixanol solution (OptiPrep) was injected to form a concentration gradient with the denser fluid at the center, see Fig. 1. General defocusing particle tracking [46] was used to record the motion of $1 \mu \mathrm{m}$-diameter polystyrene tracer beads. The fluid streaming velocity was computed by subtracting the radiation-force contribution from the bead veloicty $[22,47]$. At time $\tau=0$, the flow was stopped, and the general defocusing particle tracking measurements (10 fps) were conducted with the transducer driven at $2.0 \mathrm{~V}$ peak-topeak voltage and the frequency swept from 1.95 to $2.05 \mathrm{MHz}$ in cycles of $10 \mathrm{~ms}$ to produce a standing half wave across the width [48] with $E_{\mathrm{ac}}=52 \mathrm{~Pa}$ [49]. The frequency sweep ensures that resonance conditions (1.96 and $1.97 \mathrm{MHz}$ in pure water and in $20 \%$ iodixanol, respectively) are achieved throughout the experiment during the time evolution of the concentration field. For each set of measurements, the particle motion was recorded for $160 \mathrm{~s}$ to observe the evolution of the acoustic streaming. The experiment was repeated 16 times to improve the statistics.

Results.-Experimental data and simulation results for the acoustic streaming in the channel cross section are plotted in Fig. 2. Columnwise, the figure first shows the inhomogeneous-fluid streaming at $\tau=35 \mathrm{~s}$ and $\tau=55 \mathrm{~s}$, and then the steady homogeneous-fluid streaming. In the rows are (a) the raw experimental particle positions, (b) the grid-interpolated experimental velocity field, and (c) the simulated velocity field. The acoustically stabilized inhomogeneity distribution remains almost one dimensional with the denser fluid at the center diffusing sidewards; see the Supplemental Material [50]. Hence, recalling that for iodixanol $\boldsymbol{\nabla} \kappa_{0} \approx-\left(\rho_{0} c_{0}\right)^{-2} \nabla \rho_{0}$, we may quantify the inhomogeneity with the single parameter of the excess mass density $\hat{\rho}_{*}$ at the center relative to the sides [51]. The experimental inhomogeneous-fluid streaming pattern evolves towards the homogeneous steady state as diffusion (and, to a lesser extent, advection) diminishes the initial $\hat{\rho}_{*}$ of $10 \%$ to $4 \%$ and $2 \%$ at $\tau=35 \mathrm{~s}$ and $55 \mathrm{~s}$, respectively. We note that the time scale for the evolution of the inhomogeneous-fluid streaming $(\sim 10 \mathrm{~s})$ is orders of magnitude larger than that for achieving steady-state streaming in a homogeneous fluid $(\sim 1 \mathrm{~ms})$ [36].

Evidently, the inhomogeneous-fluid streaming is initially confined close to the boundaries and suppressed in the bulk as compared to homogeneous-fluid streaming. To quantify this suppression of streaming, we define the vortex size $\Delta$ as the orthogonal distance from the boundary to the vortex center, where $\boldsymbol{v}=\mathbf{0}$. In Fig. 3(a), the simulated vortex size $\Delta$ and the excess mass density $\hat{\rho}_{*}$ are plotted as functions of time. $\Delta$ increases slowly in time, as $\hat{\rho}_{*}$ decreases by diffusion, until a transition occurs when a critically weak inhomogeneity is reached. At this point the streaming expands into the bulk and becomes similar to homogeneous-fluid streaming. Figure 3(a) shows that $\Delta$ and $\hat{\rho}_{*}$ are inversely related, supporting the hypothesis that the inhomogeneity-induced part of $\boldsymbol{f}_{\text {ac }}$ [Eq. (9)] suppresses the boundary-driven streaming. 
(a)

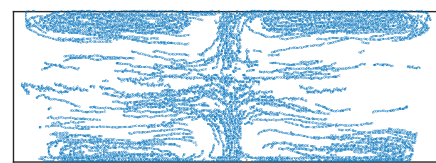

(b)

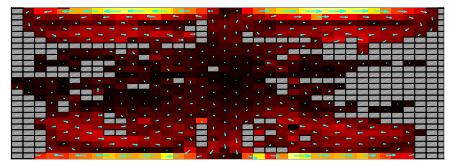

(c)

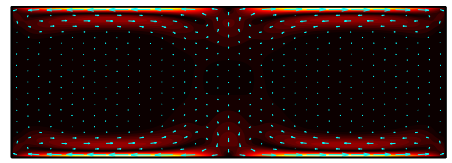

Inhomogeneous $(\tau=35 \mathrm{~s})$
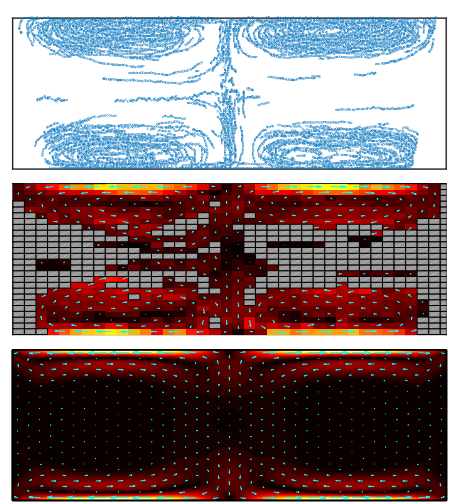

Inhomogeneous $(\tau=55 \mathrm{~s})$
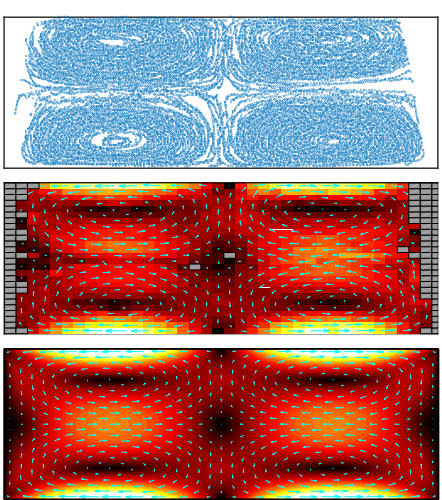

Homogeneous

FIG. 2. Acoustic streaming in the inhomogeneous fluid at $\tau=35 \mathrm{~s} \mathrm{(column} \mathrm{1)} \mathrm{and} \tau=55 \mathrm{~s}$ (column 2) and in the corresponding homogenized fluid (column 3). (a) Experimental particle positions (blue points). (b) Experimental streaming velocity amplitude $|\boldsymbol{v}|$ $(0 \mu \mathrm{m} / \mathrm{s}$, black; $35 \mu \mathrm{m} / \mathrm{s}$, white) with the arrows (cyan) indicating the direction. Spatial bins with no data points are excluded (gray).

(c) Simulated streaming velocity, same colors as in (b).

We further assess the validity of the above-mentioned hypothesis by estimating $\Delta$ from a scaling argument. In a homogeneous fluid, being interested in the bulk length scale of the flow, the only relevant length scales are the channel dimensions $H$ and $W$ (given the half-wave resonance, $\left.\lambda_{0}=2 W\right)$. In the shallow-channel limit, the explicit analytical solution yields $\Delta_{\text {hom }}=(1-1 / \sqrt{3})(H / 2)=$ $28 \mu \mathrm{m}[6]$. In a density-stratified medium, an additional relevant length scale is $L_{\rho}$ of the density gradient $\nabla \rho_{0} \approx \rho_{0} / L_{\rho}$. Writing $\rho_{0}=\rho_{0}^{(0)}[1+\hat{\rho}]$, the inhomogeneity-induced part of $f_{\text {ac }}$ [Eq. (9)] is of the order $f_{\text {ac }} \approx E_{\mathrm{ac}} \nabla \hat{\rho}$. We may then estimate $\Delta$ as the length scale on which the shear stress $\eta_{0} \nabla^{2} v_{R} \approx \eta_{0} v_{R} / \Delta^{2}$, associated with the boundary-driven Rayleigh streaming velocity amplitude $v_{R}=(3 / 2) E_{\text {ac }} \rho_{0}^{-1} c_{0}^{-1}$ [4], is balanced by $f_{\text {ac. }}$ Using the early-time values $\hat{\rho} \approx 0.1$ and $L_{\rho} \approx W / 2$, we obtain

$$
\Delta \approx \sqrt{\frac{3}{2} \frac{\nu_{0}}{c_{0}} \frac{1}{|\nabla \hat{\rho}|}} \approx \sqrt{\frac{3}{2} \frac{\nu_{0}}{c_{0}} \frac{L_{\rho}}{\hat{\rho}}} \approx 2 \mu \mathrm{m} .
$$

This estimate is an order of magnitude smaller than $\Delta_{\text {hom }}$, in good agreement with experiments and simulations. It supports the hypothesis that $\Delta \ll \Delta_{\text {hom }}$ due to the inhomogeneity-induced acoustic force density. Equation (10) furthermore illustrates why $\Delta$ grows in time; as time progresses, the inhomogeneity weakens by diffusion; i.e., $|\nabla \hat{\rho}|$ decreases, and consequently $\Delta$ grows.

The time scale characterizing the growth of the vortex size $\Delta$ towards the value $\Delta_{\text {hom }}$ is consequently set by diffusion. In the $2 \mathrm{D}$ simulation, where the diffusion is essentially $1 \mathrm{D}$ (across the width), the time scale of diffusion across one third of the channel width is $\tau_{\text {diff, } 1 \mathrm{D}}=(2 D)^{-1}(W / 3)^{2}=87 \mathrm{~s}$. Figure 3(a) shows a rapid transition in the simulated vortex size occurring around $\tau \approx 90 \mathrm{~s}$, see also the Supplemental Material [50]. However, experimentally, we find that the transition occurs earlier and less rapidly around $\tau \approx 60 \mathrm{~s}$, see Fig. 3(b). Because axial variations in the acoustic field cannot be avoided in the experiment [52], and because such variations destroy translational invariance, the axial concentration gradients tend to change the diffusion from $1 \mathrm{D}$ to $2 \mathrm{D}$, which would halve the diffusion time, $\tau_{\text {diff,2D }}=(4 D)^{-1}(W / 3)^{2}=43 \mathrm{~s}$. Most likely, the effective diffusion in the experiment is in between the idealized 1D and 2D diffusion. In Fig. 3(b), the experimental data for the vortex size $\Delta$ is plotted as a function of time $\tau$, along with the simulation result for unscaled and rescaled time for 1D and 2D diffusion, respectively. The experimental data fall mostly between the two curves, and given that there are no free fitting parameters, the agreement between theory and experiment is reasonable.

The 2D simulation successfully captures the essential physics of the experiment, including the initial suppression of streaming followed by the growth of the vortex size and

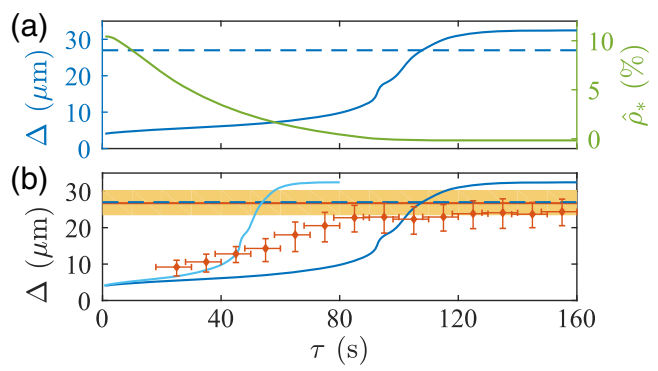

FIG. 3. (a) Simulation results for the vortex size $\Delta$ (left axis, blue curve), $\Delta_{\text {hom }}$ (left axis, dashed blue line), and the excess mass density $\hat{\rho}_{*}$ (right axis, green curve) as functions of time $\tau$. (b) Experimental results for $\Delta$ (red dots with error bars) and $\Delta_{\text {hom }}$ (red line with orange error-bar band), plotted with the simulation results for $\Delta$ (blue curve for unscaled time, light blue curve for rescaled time, see text) and $\Delta_{\text {hom }}$ (dashed blue line) as functions of time $\tau$. 
the transition to a steady state. However, Fig. 3(b) indicates that the simulation reaches a steady state that overestimates the long-time limit of $\Delta$. Interestingly, this is caused by an imperfect homogenization in 2D due to a delicate balance between advective flows and diffusive currents, leaving a slight steady-state overconcentration at the sidewalls [small negative $\hat{\rho}_{*}$, see Fig. 3(a) at $\tau=160 \mathrm{~s}$ ] that speeds up the flow in the center. Experimentally, however, the lack of perfect translational symmetry leads to homogeneous-fluid streaming at long time scales in agreement with homogeneous-fluid simulations, see Fig. 3(b).

Conclusion.-Theoretically, numerically, and experimentally, we have investigated the problem of acoustic streaming in inhomogeneous fluids with acoustically stabilized inhomogeneities. We have combined the theories of acoustic streaming and the acoustic force density, and developed a numerical model that simulates viscous inhomogeneous acoustics (the fast-time-scale dynamics) and the resulting flows due to the generalized acoustic force density (the slow-time-scale dynamics), allowing the interpretation of our microfluidic experiments with aqueous iodixanol solutions. We find that acoustic streaming is markedly different in homogeneous and inhomogeneous fluids as summarized by the main findings (i)-(iii) in the introduction. Our study is fundamental in scope, but the suppression of acoustic streaming in inhomogeneous fluids may enable ultrasound handling of nanoparticles in standard acoustophoretic chips.

We are grateful to R. Barnkob and M. Rossi, Universität der Bundeswehr München, for providing the software GDPTLab [46]. W. Q. was supported by the People Programme (Marie Curie Actions) EC-FP7/2007-2013, REA Grant No. 609405 (COFUNDPostdocDTU).

*jonkar@fysik.dtu.dk †ruus@fysik.dtu.dk

[1] T. M. Squires and S. R. Quake, Microfluidics: Fluid physics at the nanoliter scale, Rev. Mod. Phys. 77, 977 (2005).

[2] M. Wiklund, R. Green, and M. Ohlin, Acoustofluidics 14: Applications of acoustic streaming in microfluidic devices, Lab Chip 12, 2438 (2012).

[3] M. Faraday, On a peculiar class of acoustical figures; and on certain forms assumed by groups of particles upon vibrating elastic surfaces, Phil. Trans. R. Soc. London 121, 299 (1831).

[4] L. Rayleigh, On the circulation of air observed in Kundt's tubes, and on some allied acoustical problems, Phil. Trans. R. Soc. London 175, 1 (1884).

[5] H. Schlichting, Berechnung ebener periodischer grenzeschichtströmungen, Phys. Z. 33, 327 (1932).

[6] P. B. Muller, M. Rossi, A. G. Marín, R. Barnkob, P. Augustsson, T. Laurell, C. J. Kähler, and H. Bruus, Ultrasound-induced acoustophoretic motion of microparticles in three dimensions, Phys. Rev. E 88, 023006 (2013).
[7] P. Tho, R. Manasseh, and A. Ooi, Cavitation microstreaming patterns in single and multiple bubble systems, J. Fluid Mech. 576, 191 (2007).

[8] C. Eckart, Vortices and streams caused by sound waves, Phys. Rev. 73, 68 (1948).

[9] A. Riaud, M. Baudoin, O. Bou Matar, J.-L. Thomas, and P. Brunet, On the influence of viscosity and caustics on acoustic streaming in sessile droplets: An experimental and a numerical study with a cost-effective method, J. Fluid Mech. 821, 384 (2017).

[10] W. L. Nyborg, Acoustic streaming due to attenuated plane waves, J. Acoust. Soc. Am. 25, 68 (1953).

[11] W. L. Nyborg, Acoustic streaming near a boundary, J. Acoust. Soc. Am. 30, 329 (1958).

[12] J. Lighthill, Acoustic streaming, J. Sound Vibr. 61, 391 (1978).

[13] N. Riley, Steady streaming, Annu. Rev. Fluid Mech. 33, 43 (2001).

[14] G. Swift, Thermoacoustic engines, J. Acoust. Soc. Am. 84, 1145 (1988).

[15] M. Hamilton, Y. Ilinskii, and E. Zabolotskaya, Acoustic streaming generated by standing waves in two-dimensional channels of arbitrary width, J. Acoust. Soc. Am. 113, 153 (2003).

[16] M. Hamilton, Y. Ilinskii, and E. Zabolotskaya, Thermal effects on acoustic streaming in standing waves, J. Acoust. Soc. Am. 114, 3092 (2003).

[17] A. A. Doinikov and A. Bouakaz, Theoretical investigation of shear stress generated by a contrast microbubble on the cell membrane as a mechanism for sonoporation, J. Acoust. Soc. Am. 128, 11 (2010).

[18] J. Wu and W. L. Nyborg, Ultrasound, cavitation bubbles and their interaction with cells, Adv. Drug Delivery Rev. 60, 1103 (2008).

[19] P. Marmottant and S. Hilgenfeldt, Controlled vesicle deformation and lysis by single oscillating bubbles, Nature (London) 423, 153 (2003).

[20] H. Bruus, J. Dual, J. Hawkes, M. Hill, T. Laurell, J. Nilsson, S. Radel, S. Sadhal, and M. Wiklund, Forthcoming lab on a chip tutorial series on acoustofluidics: Acoustofluidicsexploiting ultrasonic standing wave forces and acoustic streaming in microfluidic systems for cell and particle manipulation, Lab Chip 11, 3579 (2011).

[21] J. Friend and L. Y. Yeo, Microscale acoustofluidics: Microfluidics driven via acoustics and ultrasonics, Rev. Mod. Phys. 83, 647 (2011).

[22] R. Barnkob, P. Augustsson, T. Laurell, and H. Bruus, Acoustic radiation- and streaming-induced microparticle velocities determined by microparticle image velocimetry in an ultrasound symmetry plane, Phys. Rev. E 86, 056307 (2012).

[23] B. Hammarström, T. Laurell, and J. Nilsson, Seed particle enabled acoustic trapping of bacteria and nanoparticles in continuous flow systems, Lab Chip 12, 4296 (2012).

[24] D. J. Collins, B. Morahan, J. Garcia-Bustos, C. Doerig, M. Plebanski, and A. Neild, Two-dimensional single-cell patterning with one cell per well driven by surface acoustic waves, Nat. Commun. 6, 8686 (2015).

[25] A. Marin, M. Rossi, B. Rallabandi, C. Wang, S. Hilgenfeldt, and C.J. Kähler, Three-Dimensional Phenomena in 
Microbubble Acoustic Streaming, Phys. Rev. Applied 3, 041001 (2015).

[26] P. Hahn, I. Leibacher, T. Baasch, and J. Dual, Numerical simulation of acoustofluidic manipulation by radiation forces and acoustic streaming for complex particles, Lab Chip 15, 4302 (2015).

[27] F. Guo, Z. Mao, Y. Chen, Z. Xie, J. P. Lata, P. Li, L. Ren, J. Liu, J. Yang, M. Dao, S. Suresh, and T. J. Huang, Threedimensional manipulation of single cells using surface acoustic waves, Proc. Natl. Acad. Sci. U.S.A. 113, 1522 (2016).

[28] S. Deshmukh, Z. Brzozka, T. Laurell, and P. Augustsson, Acoustic radiation forces at liquid interfaces impact the performance of acoustophoresis, Lab Chip 14, 3394 (2014).

[29] P. Augustsson, J. T. Karlsen, H.-W. Su, H. Bruus, and J. Voldman, Iso-acoustic focusing of cells for size-insensitive acousto-mechanical phenotyping, Nat. Commun. 7, 11556 (2016).

[30] J. T. Karlsen, P. Augustsson, and H. Bruus, Acoustic Force Density Acting on Inhomogeneous Fluids in Acoustic Fields, Phys. Rev. Lett. 117, 114504 (2016).

[31] J. T. Karlsen and H. Bruus, Acoustic Tweezing and Patterning of Concentration Fields in Microfluidics, Phys. Rev. Applied 7, 034017 (2017).

[32] G. P. Chini, Z. Malecha, and T. D. Dreeben, Large-amplitude acoustic streaming, J. Fluid Mech. 744, 329 (2014).

[33] M. Antfolk and T. Laurell, Continuous flow microfluidic separation and processing of rare cells and bioparticles found in blood-a review, Anal. Chim. Acta 965, 9 (2017).

[34] P. B. Muller, R. Barnkob, M. J. H. Jensen, and H. Bruus, A numerical study of microparticle acoustophoresis driven by acoustic radiation forces and streaming-induced drag forces, Lab Chip 12, 4617 (2012).

[35] M. Hoyos and A. Castro, Controlling the acoustic streaming by pulsed ultrasounds, Ultrasonics 53, 70 (2013).

[36] P. B. Muller and H. Bruus, Theoretical study of timedependent, ultrasound-induced acoustic streaming in microchannels, Phys. Rev. E 92, 063018 (2015).

[37] M. Antfolk, P. B. Muller, P. Augustsson, H. Bruus, and T. Laurell, Focusing of sub-micrometer particles and bacteria enabled by two-dimensional acoustophoresis, Lab Chip 14, 2791 (2014).
[38] Z. Mao, P. Li, M. Wu, H. Bachman, N. Mesyngier, X. Guo, S. Liu, F. Costanzo, and T. J. Huang, Enriching nanoparticles via acoustofluidics, ACS Nano 11, 603 (2017).

[39] D. J. Collins, Z. Ma, J. Han, and Y. Ai, Continuous microvortex-based nanoparticle manipulation via focused surface acoustic waves, Lab Chip 17, 91 (2017).

[40] P. G. Bergmann, The wave equation in a medium with a variable index of refraction, J. Acoust. Soc. Am. 17, 329 (1946).

[41] P. M. Morse and K. U. Ingard, Theoretical Acoustics (Princeton University Press, Princeton, NJ, 1986).

[42] J. Lei, P. Glynne-Jones, and M. Hill, Comparing methods for the modelling of boundary-driven streaming in acoustofluidic devices, Microfluid. Nanofluid. 21, 23 (2017).

[43] COMSOL Multiphysics 5.2, www.comsol.com, 2015.

[44] The mesh-element size grows from $\Delta h=0.1 \mu \mathrm{m}$ in the boundary layers to $\Delta h=1.3 \mu \mathrm{m}$ in the bulk. The order of the Lagrange shape functions is quadratic for $p$ and cubic for $s$ and $\boldsymbol{v}$.

[45] To fix $E_{\text {ac }}$, which varies due to small shifts in resonance frequency as $s(\boldsymbol{r}, \tau)$ evolves, we compensate by adjusting the sidewall actuation amplitude $d_{0}(\tau)$ iteratively, see also Ref. [34].

[46] R. Barnkob, C. J. Kahler, and M. Rossi, General defocusing particle tracking, Lab Chip 15, 3556 (2015).

[47] J. T. Karlsen and H. Bruus, Forces acting on a small particle in an acoustical field in a thermoviscous fluid, Phys. Rev. E 92, 043010 (2015).

[48] O. Manneberg, B. Vanherberghen, B. Onfelt, and M. Wiklund, Flow-free transport of cells in microchannels by frequency-modulated ultrasound, Lab Chip 9, 833 (2009).

[49] We estimate $E_{\mathrm{ac}}=\left(E_{\mathrm{ac}}^{20 \%}+E_{\mathrm{ac}}^{0 \%}\right) / 2$, where $E_{\mathrm{ac}}^{20 \%}$ and $E_{\mathrm{ac}}^{0 \%}$ are measured in homogeneous $20 \%$ and $0 \%$ iodixanol solutions.

[50] See Supplemental Material at http://link.aps.org/ supplemental/10.1103/PhysRevLett.120.054501 for simulation results of density evolution and particle trajectories.

[51] $\hat{\rho}_{*}=\rho_{0}^{\text {center }} / \rho_{0}^{\text {sidewall }}-1$, evaluated numerically at midheight.

[52] P. Augustsson, R. Barnkob, S. T. Wereley, H. Bruus, and T. Laurell, Automated and temperature-controlled micropiv measurements enabling long-term-stable microchannel acoustophoresis characterization, Lab Chip 11, 4152 (2011). 